\title{
The SafeBoosC II randomized trial: treatment guided by near-infrared spectroscopy reduces cerebral hypoxia without changing early biomarkers of brain injury
}

\author{
Anne M. Plomgaard', Wim van Oeveren², Tue H. Petersen ${ }^{3}$, Thomas Alderliesten ${ }^{4}$, Topun Austin ${ }^{5}$, Frank van Bel ${ }^{4}$, \\ Manon Benders ${ }^{4}$, Olivier Claris ${ }^{6}$, Eugene Dempsey ${ }^{7}$, Axel Franz ${ }^{8}$, Monica Fumagalli ${ }^{9}$, Christian Gluud $^{10}$, Cornelia Hagmann ${ }^{11}$, \\ Simon Hyttel-Sorensen ${ }^{1}$, Petra Lemmers ${ }^{4}$, Adelina Pellicer ${ }^{12}$, Gerhard Pichler ${ }^{13}$, Per Winkel ${ }^{10}$ and Gorm Greisen ${ }^{1}$
}

\begin{abstract}
BACKGROUND: The SafeBoosC phase II multicentre randomized clinical trial investigated the benefits and harms of monitoring cerebral oxygenation by near-infrared spectroscopy (NIRS) combined with an evidence-based treatment guideline vs. no NIRS data and treatment as usual in the control group during the first $72 \mathrm{~h}$ of life. The trial demonstrated a significant reduction in the burden of cerebral hypoxia in the experimental group. We now report the blindly assessed and analyzed treatment effects on electroencephalographic (EEG) outcomes (burst rate and spectral edge frequency 95\% (SEF95)) and blood biomarkers of brain injury (S100 $\beta$, brain fatty acid-binding protein, and neuroketal).
\end{abstract}

METHODS: One hundred and sixty-six extremely preterm infants were randomized to either experimental or control group. EEG was recorded at $64 \mathrm{~h}$ of age and blood samples were collected at 6 and $64 \mathrm{~h}$ of age.

RESULTS: One hundred and thirty-three EEGs were evaluated. The two groups did not differ regarding burst rates (experimental 7.2 vs. control 7.7 burst/min) or SEF95 (experimental 18.1 vs. control $18.0 \mathrm{~Hz}$ ). The two groups did not differ regarding blood $\mathbf{S 1 0 0} \beta$, brain fatty acid-binding protein, and neuroketal concentrations at 6 and $64 \mathrm{~h}$ ( $n=123$ participants).

CONCLUSION: Treatment guided by NIRS reduced the cerebral burden of hypoxia without affecting EEG or the selected blood biomarkers.

$\mathbf{T}$ he mortality in extremely preterm infants is approximately $25 \%$ and the prevalence of moderate and severe neurodevelopmental impairment in the surviving infants is as high as $25 \%$ (1). The etiology behind this neurodevelopmental impairment is multifactorial and partly related to the immaturity of the respiratory and circulatory systems leading to episodes of cerebral hypoxia. Cerebral hypoxia, in turn, through complex interactions between destructive and developmental disturbances may lead to micro- and macroscopic structural brain damage (2). The outcome of relevance to the individual child is neurodevelopmental impairment and possible ensuing disabilities, which cannot be determined before the child is fully grown. Therefore, there is a need for identifying early surrogate outcomes. Long before the age of reliable testing of neurological functions or intelligence, it is possible to visualize brain injuries such as intraventricular and parenchymal hemorrhage by early cranial ultrasound (3). Later cranial ultrasound may show ventricular dilatation as a sign of exvacuo dilatation caused by cerebral atrophy, or periventricular leukomalacia $(2,4,5)$, whereas magnetic resonance imaging at term equivalent age may show minor degrees of white matter damage (3). Even before structural brain damage is visible, there are other means of detecting injury to the brain of the extremely preterm infant. Several cohort studies have demonstrated good correlations between early electroencephalography (EEG) and later developmental outcomes (6,7). A number of biomarkers show correlation with brain injury. Blood S100 $\beta$ is elevated in newborn infants with intraventricular hemorrhage (8) and hypoxic-ischemic encephalopathy (9). Blood brain fatty acidbinding protein (BFABP) is elevated in elderly patients with neurodegenerative diseases (10) and cerebrospinal fluid neuroketal is elevated in preterm infants with white matter damage on magnetic resonance imaging (11).

The SafeBoosC phase II randomized trial demonstrated that the combination of cerebral oxygenation monitoring by near-infrared spectroscopy (NIRS) combined with an evidence-based treatment guideline (12) vs. blinded collection of cerebral NIRS data and treatment as usual for $72 \mathrm{~h}$ significantly reduced the burden of cerebral hypoxia (13). To investigate the

\footnotetext{
'Department of Neonatology, Rigshospitalet, Copenhagen University Hospital, Copenhagen, Denmark; ${ }^{2}$ HaemoScan B.V., Groningen, The Netherlands; ${ }^{3}$ Research Unit on Brain Injury Neurorehabilitation Copenhagen, Department of Neurorehabilitation, TBI Unit, Rigshospitalet, Copenhagen University Hospital, Hvidovre, Denmark; ${ }^{4}$ Wilhelmina Children's Hospital, University Medical Center Utrecht, Utrecht, The Netherlands; ${ }^{5}$ Rosie Maternity Hospital Cambridge University Hospitals NHS Foundation Trust, Cambridge, UK; ${ }^{6}$ Department of Neonatology, Hospital Femme Mere Enfants, Bron, France; ${ }^{7}$ INFANT Centre, University College Cork, Cork, Ireland; ${ }^{8}$ Department of Neonatology, University of Tuebingen, Tübingen, Germany; ${ }^{9} \mathrm{NICU}$, Fondazione IRCCS Ca' Granda Ospedale Maggiore Policlinico, Milan, Italy; ${ }^{10} \mathrm{Copenhagen} \mathrm{Trial} \mathrm{Unit,} \mathrm{Centre} \mathrm{for} \mathrm{Clinical} \mathrm{Intervention}$ Research, Rigshospitalet, Copenhagen University Hospital, Copenhagen, Denmark; ${ }^{11}$ Department of Neonatology, University of Zurich, Zurich, Switzerland; ${ }^{12}$ Department of Neonatology, La Paz University Hospital, Madrid, Spain; ${ }^{13}$ Department of Pediatrics, Medical University of Graz, Graz, Austria. Correspondence: Anne Mette Plomgaard (amplomgaard@gmail.com) 
Table 1. Entry variables and 72-h outcomes of the included infants

\begin{tabular}{|c|c|c|c|c|}
\hline & \multicolumn{2}{|c|}{ Infants with EEG } & \multicolumn{2}{|c|}{ Infants with blood samples at 6 and $64 \mathrm{~h}$} \\
\hline & $\begin{array}{l}\text { Experimental } \\
\text { group }(n=68)\end{array}$ & $\begin{array}{l}\text { Control group } \\
\quad(n=65)\end{array}$ & $\begin{array}{l}\text { Experimental } \\
\text { group }(n=66)\end{array}$ & $\begin{array}{l}\text { Control group } \\
\quad(n=58)\end{array}$ \\
\hline Birth weight (g), median (range) & $821(410-1,286)$ & $880(490-1,330)$ & $772(410-1,286)$ & $880(515-1,330)$ \\
\hline GA (wk), median (IQR) & $26.6(25.8-27.4)$ & $27.0(25.7-27.6)$ & $26.6(24.4-27.3)$ & $27.0(25.7-27.6)$ \\
\hline Apgar below 5 at $5 \min , n(\%)$ & $10(15)$ & $9(14)$ & $12(18)$ & $8(14)$ \\
\hline Antenatal steroids (complete course) & $44(65)$ & $48(74)$ & $47(71)$ & $41(71)$ \\
\hline \multicolumn{5}{|l|}{ Outcomes at $72 \mathrm{~h}$} \\
\hline Burden of hyperoxia (\%hours), median (IQR) & $1.2(0.4-11.8)$ & $4(0.2-29.5)$ & $1.0(0.3-6.2)$ & $1.8(0.2-21.3)$ \\
\hline
\end{tabular}

possible benefits of the experimental intervention on putative surrogate outcomes (14-16), we recorded EEG with amplitudeintegrated EEG tracing at the end of the intervention. We also analyzed the blood taken at the beginning and at the end of the intervention for the brain injury molecular biomarkers $S 100 \beta$, BFABP, and neuroketal. We hypothesized that the intervention would reduce the interburst interval (IBI) (6), which was a secondary outcome in the trial. Furthermore, an increased EEG spectral edge frequency 95\% (SEF95) (17) and decreased levels of the brain injury molecular biomarkers S100 $\beta$, BFABP, and neuroketal (exploratory outcomes) were expected.

\section{RESULTS}

\section{Infant Characteristics}

Patient characteristics at entry were similar across intervention groups but the burden of cerebral hypoxia was significantly lower at the end of intervention ( $72 \mathrm{~h}$ of age) in the experimental group vs. the control group (Table 1). There were no differences in GA and birth weight for infants with and without EEG or with or without molecular biomarkers at the two time points, although more infants without EEG had an Apgar score below five points at $5 \mathrm{~min}$.

\section{Electroencephalography}

Eight infants died before $64 \mathrm{~h}$ of age (three in the experimental group and five in the control group) and another eight infants did not have any EEG recordings. Thus, one hundred and fifty EEGs were recorded. Seventeen of the recorded EEGs were not evaluable, which include impossible to read $(n=7)$, too short $(n=2)$, no artifact-free epochs $(n=7)$, or the infant was too old at the time of the EEG recording $(n=1)$. In total, we therefore analyzed EEGs from 133 infants. Data were collected using eight different EEG monitors: Micromed EEG system (Mogliano, Veneta, Italy) $(n=29)$; Nervus monitor (Cephalon, Norresundby, Denmark) $(n=29)$; Olympic cerebral function monitor (Natus, Pleasanton, CA) $(n=29)$; BRM2 and BRM3 (Natus, former Brainz monitor) ( $n=16$ and $n=3)$; NicoletOne
Table 2. EEG recording details and results

\begin{tabular}{|c|c|c|c|}
\hline EEG recording details & $\begin{array}{l}\text { Experimental } \\
\text { group }(n=68)\end{array}$ & $\begin{array}{l}\text { Control group } \\
\quad(n=65)\end{array}$ & $P$ value \\
\hline Age at EEG (h), median (IQR) & $64.8(62.7-68.5)$ & $65.8(62.4-68.4)$ & NS \\
\hline Epochs per infant, median (IQR) & $12(11-16)$ & $12(11-20)$ & NS \\
\hline $\begin{array}{l}\text { Sampling frequency } 256 \mathrm{~Hz} \text {, } \\
n(\%)\end{array}$ & $54(79)$ & $50(77)$ & $\mathrm{NS}^{\mathrm{a}}$ \\
\hline $\begin{array}{l}\text { Sampling frequency } 200 \mathrm{~Hz} \text {, } \\
n(\%)\end{array}$ & $14(21)$ & $15(23)$ & \\
\hline Data filtered at 2-15 Hz, $n$ (\%) & $13(20)$ & $10(16)$ & NS \\
\hline Opioid, $n(\%)$ & $16(24)$ & $21(32)$ & NS \\
\hline Sedative, $n(\%)$ & $9(13)$ & $5(8)$ & NS \\
\hline \multicolumn{4}{|l|}{ EEG results } \\
\hline Severe burst suppression & $3(4)$ & $2(3)$ & \\
\hline Burst suppression & $11(16)$ & $7(11)$ & $0.75^{\mathrm{b}}$ \\
\hline Discontinuous & $41(60)$ & $41(63)$ & \\
\hline Continuous & $13(20)$ & $15(23)$ & \\
\hline $\begin{array}{l}\text { Burst rate (bursts/min) } \\
\text { mean }(\mathrm{SE})^{\mathrm{c}}\end{array}$ & $7.2(0.72)$ & $7.7(0.73)$ & 0.51 \\
\hline $\begin{array}{l}\text { Spectral edge frequency } \\
95 \%(\mathrm{~Hz}) \text {, mean }(\mathrm{SE})^{c}\end{array}$ & $18.1(0.10)$ & $18.0(0.10)$ & 0.51 \\
\hline
\end{tabular}

IQR, inter quartile range; $\mathrm{SE}$, standard error; NS, nonsignificant; $\mathrm{EEG}$, electroencephalography.

${ }^{a}$ Chi-squared test of distribution of the sampling frequency between experimental and control. ${ }^{\circ} \mathrm{C}$ Chi-squared test of distribution of the rEEG classification between experimental and control. 'Result of mixed modelling after random exclusion of one twin from each twin pair.

video-EEG system (Carefusion, Madison, WI) $(n=16)$; g.recorder (g-tec, Graz, Austria) $(n=7)$; and Moberg CNS monitor (Moberg Research, Ambler, PA) $(n=4)$. The median age at the time of EEG recording, number of sedated infants, and the number of infants treated with opioid did not differ between the intervention groups (Table 2). The interobserver 
agreement for the artifact rejection was $92 \%$ and the Kappa value was 0.61 .

After inverse transformation, the IBIs were normally distributed and by multiplication by $60 \mathrm{~s} / \mathrm{min}$ expressed as burst rate (bursts/min). Inter cluster correlations (ICCs) were above 0.15 for both burst rate and SEF95. Therefore, the between-groups analyses were conducted after randomly excluding one of each twin pair. Univariate analysis for burst rate and SEF95 was unaffected by GA $(P=0.42$ and $P=0.40)$. The burst rate was significantly affected by EEG filter $(P<0.0001)$, type of EEG device $(P=0.045)$, and opioid treatment $(P=0.004)$. SEF95 was significantly affected by EEG sampling rate $(P<0.0001)$ and type of EEG device $(P<0.0001)$. Type of EEG device and EEG filter and type of EEG device and EEG sampling rate were strongly correlated; therefore, only EEG filter and EEG sampling rate, opioid treatment, and GA were retained in the multiple regression models. In multiple regression, the mean (SE) burst rate did not differ between the two intervention groups: experimental 7.2 bursts/min (0.72) vs. control 7.7 bursts/ min (0.73). The mean SEF95 (SE) did not differ between the intervention groups: experimental $18.1 \mathrm{~Hz}(0.10)$ vs. control $18.0 \mathrm{~Hz}(0.10)$ (Table 2). The delta-, theta-, alpha-, and betapower bands did not differ between the intervention groups. The raw burst rates and SEF95 separated by EEG filter and EEG sampling rate are illustrated in Figure 1a,b. The unadjusted burst rate (SD) was reduced in infants with intraventricular hemorrhage grade III or periventricular hemorrhagic infraction on day 1-4 $(n=10): 5.6$ bursts/min (3.6) compared to 9.8 bursts/min $(5.0)$ in the remaining infants $(n=120)$.

\section{Blood Biomarkers}

One hundred and twenty-three infants (66 in the experimental group vs. 57 in the control group) had blood samples taken at both the time points ( 6 and $64 \mathrm{~h}$ of age). The concentrations of the three biomarkers in the blood did not differ between the intervention groups neither at 6 nor at $64 \mathrm{~h}$ of age (Table 3 ). The main effect of the intervention is illustrated in Figure 2.

The ICC between the twins was above 0.15 for all blood biomarkers. Therefore, all analyses were conducted after the exclusion of one random twin from each twin pair. The mixedmodel analysis or nonparametric analysis, in case of nonnormal distribution of the data, confirmed the findings above (Table 3). The blood biomarkers were similar in infants with and without intraventricular hemorrhage degree III or periventricular hemorrhagic infractions.

\section{DISCUSSION}

The reduction in the burden of cerebral hypoxia in the group receiving cerebral NIRS monitoring combined with an evidence-based treatment guideline was not reflected in any of the early biomarkers of brain injury that were assessed in the SafeBoosC II trial.

\section{The Randomized Clinical Trial Design}

The SafeBoosC phase II trial was a randomized clinical multicentre trial. We blindly evaluated and analyzed EEGs in $84 \%$ $(133 / 158)$ and blood samples in $78 \%(123 / 158)$ of the infants alive at the time of the scheduled EEG recording and blood sampling. To avoid biased regression coefficients (18), we randomly excluded one of each twin pair, if the ICC was above 0.15 .

\section{The Challenges of EEG Recording}

The EEGs were recorded with eight different devices. We therefore used a multiple regression model with corrections for EEG sampling specifications (EEG filter and EEG sampling rate). The difference in SEF95 given the different sampling rates of the EEG devices was not surprising since this was linked to the differences in frequency resolution of the Fast Fourier Transform given the different sampling rates. Some of the EEG
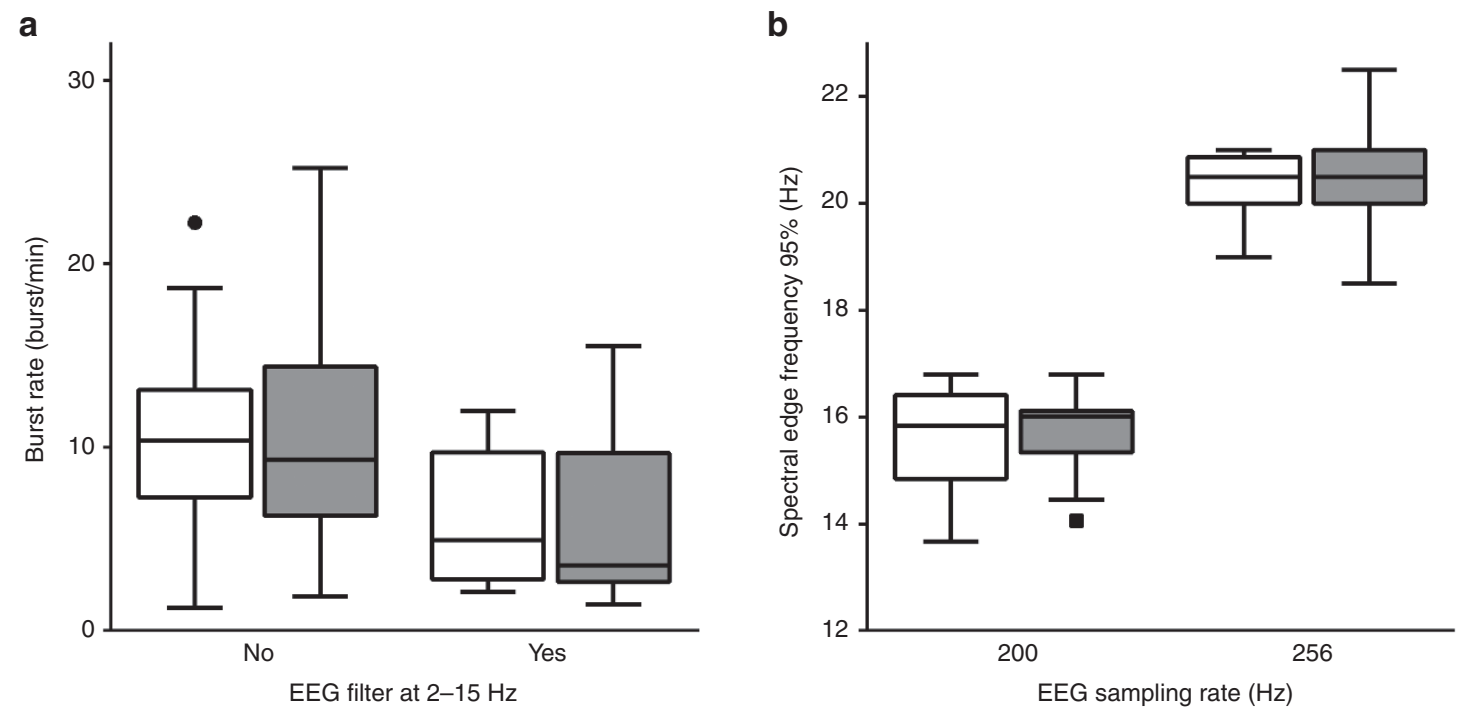

Figure 1. Intervention effects on EEG outcomes. (a) Intervention effect on burst rates, according to EEG filter of the device. (b) Intervention effect on spectral edge frequency $95 \%$, according to the EEG sampling rate of the device. $\square=$ control group, $\boldsymbol{\square}=$ experimental group. 
Table 3. Molecular brain injury biomarkers

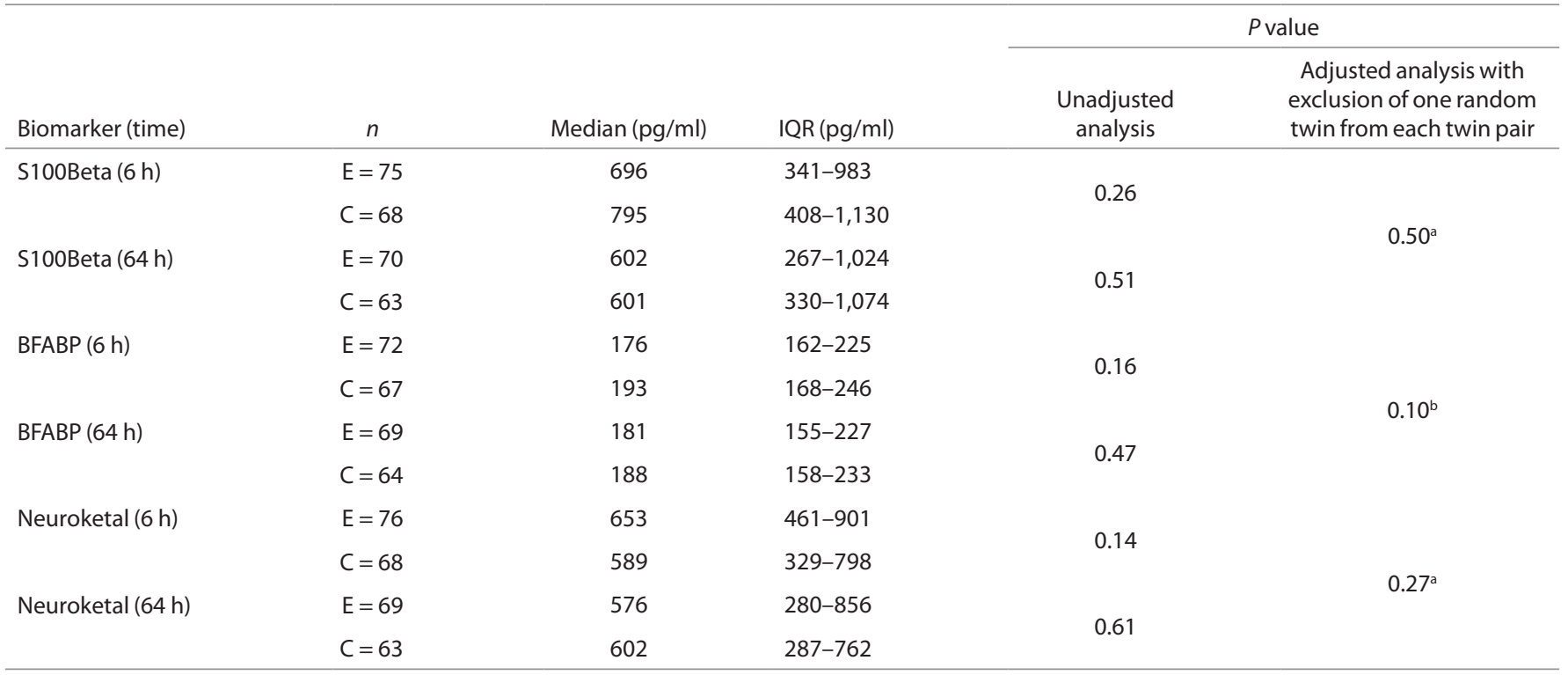

The effect of the intervention at the 6 and 64 -h time points in the middle column and the effect on the change over time to the right.

E, experimental group; C, control group; IQR, inter quartile range.

aparametric statistics. ${ }^{\text {} N o n p a r a m e t r i c ~ s t a t i s t i c s . ~}$

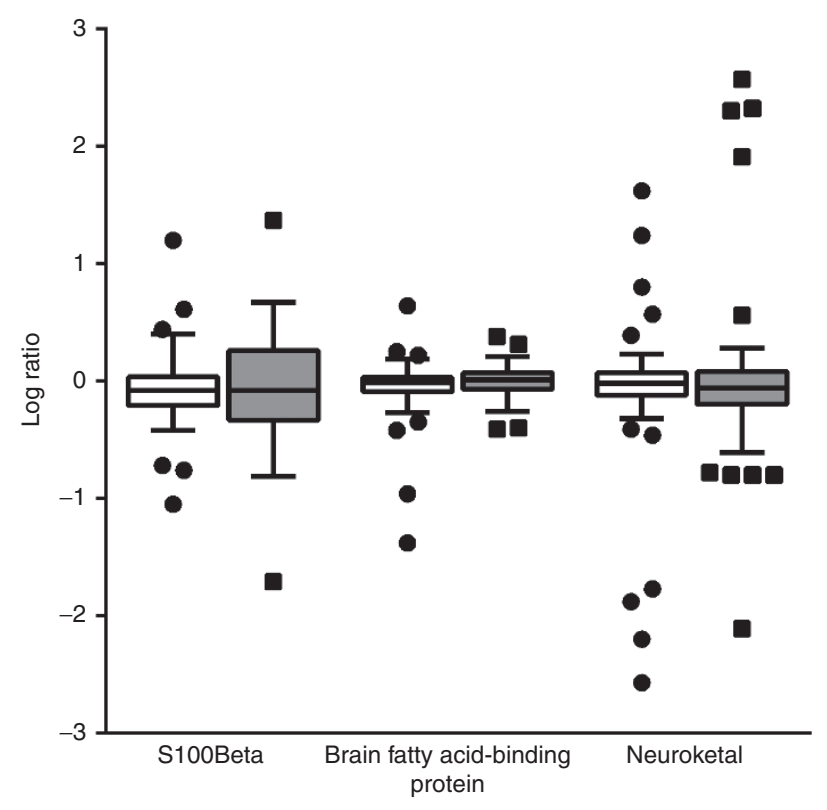

Figure 2. Intervention effect on the blood biomarkers illustrated as log ratio for the change in the concentration of the blood biomarkers from 6 to $64 \mathrm{~h}$ of age. $\square=$ control group, $\boldsymbol{\square}=$ experimental group.

amplifiers used were equipped with a band pass filter from 2 to $15 \mathrm{~Hz}$. This clearly affected the detection of burst rates since the algorithm used for this was based on co-occurrence of slow- and high-frequency oscillations (19). Nevertheless, the burst rates in the SafeBoosC II trial were in line with previously published results in this group of infants $(6,20,21)$ and the IBIs were longer in preterm infants with intraventricular hemorrhage grade III or periventricular hemorrhagic infractions, which has also previously been observed (22). However, we did not find a correlation between IBI and GA above or below $26 \mathrm{wk}$; this could be explained by the limited range of GA in the SafeBoosC trial. We therefore believe that the EEG data are robust and the results are reliable. While it is feasible to use EEG as an outcome in a multicentre randomized clinical trial, it should be realized that working with different EEG devices may complicate the analyses by adding heterogeneity.

\section{The Relevance of the Chosen Biomarkers}

EEG. In term newborn infants, severe hypoxic-ischemic brain injury is associated with EEG burst suppression, lasting hours to days and predicting later neurodevelopmental outcome and death $(23,24)$. EEG burst rate is reduced in preterm infants when arterial $\mathrm{P}_{\mathrm{CO}_{2}}$ and blood glucose concentrations are outside the normal range (20). Furthermore in preterm infants, permissive hypercapnia has been associated with increased power in the delta band of the EEG (25). Therefore, we hypothesized that EEG would be a sensitive surrogate marker of any clinically relevant brain effect of the reduction in cerebral hypoxia or hyperoxia that was aimed for in the SafeBoosC II trial. While the effect on cerebral hyperoxia was small and statistically insignificant, the reduction of cerebral hypoxia was highly statistically significant between the intervention groups, as well as potentially clinically relevant-amounting to more than a $50 \%$ reduction in cerebral hypoxia (13). We therefore expected the EEG to be different in the intervention groups. Furthermore, our present analyses are not able to determine if IBI or SEF95 is able to predict the long-term cerebral outcome of preterm infants. Several studies have investigated the predictive value of different EEG variables and later neurodevelopmental outcome, although the variables and the timing of the EEG recording that have been used differ $(6,7,22)$, and therefore EEG should not yet be discarded 
as a potential surrogate outcome of randomized clinical trials in preterm infants (14). A systematic review aiming to clarify the predictive value of EEG as a surrogate outcome for the developmental outcome in this group of infants is currently being conducted (26). We could not implement continuous EEG monitoring in this complex, multicentre SafeBoosC II trial and the age of $64 \mathrm{~h}$ was chosen as we hypothesized that the end of the intervention was the time with the highest likelihood of detecting an accumulated effect of a reduction of cerebral hypo- or hyperoxia in the experimental groupshould such be found. This approach was chosen by extrapolation from term infants with severe hypoxic-ischemic brain injury in whom the EEG effects last some days (27).

We chose burst rate and SEF as the variables for the EEG analyses as these are the most commonly used in preterm infants and have been related to long-term outcomes $(6,7,17)$. Certainly, other more sophisticated analytical methods could be used and may be more sensitive to subtle effects of moderate cerebral hypoxia (28).

Blood biomarkers. The three blood biomarkers of brain injury were explorative outcomes of the SafeBoosC II trial and the reduction in the burden of cerebral hypoxia was not reflected in any of them.

$\mathrm{S} 100 \beta$ is the best known of the three brain injury blood biomarkers and is routinely used in the screening of patients with mild to moderate head trauma (29). It is a small calcium-binding protein present in high concentration in Schwann cells and astrocytes (30) and after severe cerebral damage, it leaks from the tissue into the systemic circulation.

A small study in preterm infants found that the level of $S 100 \beta$ in blood is correlated to the degree of intraventricular hemorrhage (31), whereas a recent larger study showed no correlation (32). The level of $S 100 \beta$ is increased in term asphyxiated infants and the concentrations have been correlated to the degree of hypoxic-ischemic encephalopathy (9). Whereas hypoxic encephalopathy in term infants typically is the result of a terminal, short and severe hypoxia, the preterm infants in the SafeBoosC II trial were more likely exposed to various grades of cerebral hypoxia for shorter or longer periods during the first $72 \mathrm{~h}$ of life. There was no difference in $\mathrm{S} 100 \beta$ between the two intervention groups. This could be because the burden of cerebral hypoxia-even though the reduction in the experimental group was highly significant-was insufficient for the release of the intracellular S100 $\beta$ into the systemic circulation. Negative correlations between NIRS levels and S100 $\beta$ in critically ill children have been shown previously (33).

BFABP is a small molecule $(15 \mathrm{kDa})$, which is rapidly excreted by the kidneys without cleavage. It is released from astrocytes as response to mechanical damage, ischemia, and oxidative brain damage (34). BFABP has not been investigated previously in extremely preterm infants, but is known to be elevated in patients with various neurodegenerative diseases (10). In adult patients with acute ischemic stroke, BFABP is elevated from 2 to $3 \mathrm{~h}$ after the event and remains increased for at least $5 \mathrm{~d}$ (34). There was no difference in the level of
BFABP between the two intervention groups in the SafeBoosC II trial, which again could be explained by the insufficiency of a moderate global cerebral hypoxic exposure-compared to stroke patients experiencing an acute and severe local cerebral hypoxia.

Neuroketal concentrations in cerebrospinal fluid have been reported to be increased in preterm infants with cerebral white matter damage on magnetic resonance imaging (11). Neuroketals are compounds produced by free radical-induced peroxidation of docosahexenoic acid, which is solely present in the brain and especially vulnerable to oxidative stress (35). As the burden of hyperoxia was unaffected by the intervention in the SafeBoosC II trial, it may not be surprising that the neuroketal was the same in the two groups.

We did not observe any significant effects of cerebral oxygenation monitoring on blood levels of S100 $\beta$, BFABP, or neuroketal. Our present analyses are not able to decide if these blood biomarkers are predictive of long-term cerebral outcome in this population. Accordingly, we do not yet know if these blood biomarkers are valid surrogate outcomes for cerebral damage in relation to NIRS (14-16).

\section{Limitations of the Trial}

We did not measure the $\mathrm{P}_{\mathrm{CO}_{2}}$ in the SafeBoosC II infants, but all other physiological variables were comparable in the two intervention groups (fractional inspired oxygen, peripheral capillary oxygen saturation, mean airway pressure, heart rate, and arterial blood pressure) (13). The incidence of seizures in this population is high, however, the burden of the seizure is low; exceeding $90 \mathrm{~s}$ during $24 \mathrm{~h}$ of recording on day 3 in only $3 \%$ of the infants and thus only amounts to less than $0.2 \%$ of recording time (36). In this study, we did not investigate the seizure activity. The blood biomarker and EEG outcomes are unvalidated short-term surrogate outcomes of the intervention and assessment of neurodevelopmental outcome at $2 \mathrm{y}$ of age in this cohort is ongoing. This may enable the validation of these surrogates (14-16).

Our results do not support the hypothesis that the SafeBoosC II NIRS intervention would be effective in reducing brain injury. First, the chosen surrogate outcomes may not be valid. EEG and the blood biomarkers are not established markers for brain injury in preterm infants. However, we examined them as prime candidates and therefore consider the absence of any difference between the groups as an important signal. Is it possible that the reduced burden of cerebral hypoxia was irrelevant for the brain? Although the "burden of cerebral hypoxia" - the accumulated area under the curve expressed in \%hours-amounted to more than 200 \%hours in some infants, the threshold used as the alarm limit for intervention and for the calculation of the burden was 55\% (13). In piglet experiments at term, cerebral oxygenation has to be kept below 30\% before severe abnormalities occur in the EEG $(37,38)$ and below $35 \%$ for several hours to induce brain injury (39). Furthermore, preterm infants are normally exposed to arterial oxygen saturation of $70 \%$ or less in utero, and some neonatal units in the past (1990s) targeted arterial saturation 
at low ranges and thereby reduced the risk of retinopathy of prematurity, without increasing the risk of cerebral palsy (40), though it has later been shown that low levels of arterial saturation increase mortality (41). Therefore, our results raise the possibility that cerebral hypoxia at the levels that are common in extremely preterm infants during the first days after birth is really not significant as an etiology for brain (tissue) injury as detectable by EEG and the selected molecular biomarkers at $64 \mathrm{~h}$ of age.

\section{Conclusions}

Whereas cerebral hypoxia was reduced to less than half in the experimental group, the result was neither reflected in the EEG, nor in the three assessed blood biomarkers.

\section{METHODS \\ Study Design and Patient Characteristics}

The SafeBoosC II randomized clinical trial is a multicentre, blinded, feasibility medical device trial (42). One hundred and sixty-six extremely preterm infants from eight European countries each represented by one NICU were included (1 June 2012-31 December 2013) (13). The infants were randomized to the experimental group (cerebral NIRS monitoring with visible cerebral oxygenation levels combined with an evidence-based treatment guideline (12) listing possible interventions if the cerebral oxygenation was out of range: 55-85\%) vs. control group (blinded collection of cerebral oxygenation levels combined with treatment as usual). In both intervention groups, the NIRS monitoring was started within $3 \mathrm{~h}$ after birth and ended at $72 \mathrm{~h}$ of life. The trial is registered at ClinicalTrial.gov, NCT01590316; the protocol is available in full at http://www.safeboosc.eu.

\section{The EEG Recording}

Recording was specified in a standard operating procedure. At postnatal age of $64 \mathrm{~h}( \pm 8)$ at least $120 \mathrm{~min}$ of good quality EEG with amplitude-integrated EEG tracing was recorded. Electrodes were placed at $\mathrm{P} 3$ and P4 positions according to the international 10-20 system. Needle, disc, or hydrogel electrodes could be used according to local practice and additional electrodes were allowed. The electrode impedance was less than $20 \mathrm{k} \Omega$ during the recording. If the child was treated with morphine, other opioids, or sedative medications this was noted in an electronic record form. The raw EEG data were anonymized and uploaded for central analysis via a file transfer protocol.

\section{EEG Analysis}

All EEG analysis was performed in Matlab version R2014b (MathWorks, Natick, MA).

Artifact rejection. The raw EEG was band pass filtered $(0.5-30 \mathrm{~Hz})$ using a zero-phase filter. Since the amplitude-integrated EEG trace was missing in some infants, the filtered EEG data were converted into range EEG (rEEG) (43) to enable a standardized artifact rejection. The rEEGs were split in 10-min epochs for visual inspection. Artifacts in the rEEG epochs were independently visually identified by two of the authors (GG and AMP) blinded to treatment allocation and the clinical history of the infant. Kappa statistics was used to calculate the level of agreement. For epochs with disagreements, the raw EEG was reviewed and the epoch rejected if the presence of artifacts was confirmed.

Classification of rEEG. The rEEGs were visually classified into four categories: (i) severe burst suppression; (ii) burst suppression (mainly flat lower margin); (iii) discontinuous (lower margin below $25 \mu \mathrm{V}$ most of the time); or (iiii) continuous (lower margin above $25 \mu \mathrm{V}$ ).

IBIs were measured as the time (in seconds) between bursts of nested (high-frequency) oscillations within large slow-wave depolarizations using an extraction algorithm based on the co-occurrence of a slow $(0.5-2 \mathrm{~Hz})$ wave and higher $(8-22 \mathrm{~Hz})$ frequency oscillation. Consecutive events that occurred within $0.5 \mathrm{~s}$ of one another were counted as one and events of duration less than $4 / 22$ of a second were discounted (19). IBIs were calculated for each of the artifact-free 10 -min epochs and expressed as the median of all IBIs in all included epochs.

Spectral analysis. For each artifact-free 10-min epoch spectral analyses were conducted using Matlab routines (Neurospec 2.0, Neurospec. org). The EEG data were segmented into epochs of $2 \mathrm{~s}$ with an overlap of $50 \%$ (1s). After Fast Fourier Transformation, the spectrum was subdivided into frequency bands: delta $(0.5-4 \mathrm{~Hz})$, theta $(4.5-8 \mathrm{~Hz})$, alpha $(8.5-13 \mathrm{~Hz})$, and beta $(13.5-30 \mathrm{~Hz})$. The spectral distribution was calculated as the square root of the power in each band and expressed as percentages adding up to $100 \%$. A grand mean of the spectral distribution for each infant was calculated as the mean of all epochs.

SEF95 for each infant was defined as the frequency between 0.5 and $30 \mathrm{~Hz}$, below which 95\% of the power was present. SEF95 is expressed as a mean of all epochs.

\section{Brain Injury Biomarkers in Blood}

Blood sampling, plasma storage and analyses were specified in a standard operating procedure. At the age of $6 \mathrm{~h}( \pm 1)$ and $64 \mathrm{~h}( \pm 1), 1 \mathrm{ml}$ of blood was collected. A blood sample was only taken if the infant had an indwelling arterial or venous line. The blood was sampled in heparin tubes and centrifuged (1200×g for $12 \mathrm{~min}$ ) before the plasma was transferred to a small screw-cap tube. The samples were kept at -40 to $-20^{\circ} \mathrm{C}$ for a maximum of $1 \mathrm{wk}$, thereafter at $-80^{\circ} \mathrm{C}\left( \pm 10^{\circ} \mathrm{C}\right)$ for prolonged storage. After inclusion of the last patient, the samples were sent on dry ice to a central laboratory (HaemoScan, Groningen, The Netherlands).

\section{Laboratory Analysis}

The blood samples were analyzed for the three different brain injury biomarkers. The laboratory technician was unaware of the medical history and allocation of the infant. $S 100 \beta(50 \mu)$ was assessed by ELISA. Clone 1B2 monoclonal antibody was used as capture antibody (Abnova, Taipei, Taiwan) and biotinylated clone 8B10 as detection antibody (Hytest, Turku, Finland). Intra-assay variance is $4.6 \%$ and the lower level of quantification (LLOQ) is $39 \mathrm{pg} / \mathrm{ml}$. BFABP $(50 \mu \mathrm{l}$ ) was determined by means of ELISA with BFABP polyclonal capture antibodies and monoclonal detection antibody (HaemoScan). Intraassay variance is $6.4 \%$ and the lower level of quantification is $150 \mathrm{pg} /$ $\mathrm{ml}$. Neuroketal $(60 \mu \mathrm{l})$ determination was performed by competitive enzyme immunoassay based on purified rabbit antibodies against $\mathrm{KLH}$ complexed neuroketal (Haemoscan). Intra-assay variance is $10 \%$ and the lower level of quantification is $4.1 \mathrm{pg} / \mathrm{ml}$.

\section{Ethics}

The SafeBoosC phase II trial was approved by each hospital's research ethics committee (Hopital Femme Mere Enfants, Lyon, France; Rigshospitalet, Copenhagen, Denmark; La Paz University Hospital, Madrid, Spain; Cork University Maternity Hospital, Cork, Ireland; Wilhelmina Children's Hospital, Utrecht, The Netherlands; Medical University of Graz, Graz, Austria; Fondazione IRCCS Ca' Granda Ospedale Maggiore Policlinico, Milan, Italy; and Rosie Hospital, Cambridge University Hospitals, United Kingdom), and where required (Austria, Denmark, and France) by the competent authority responsible for medical devices. Written informed parental consent was mandatory before inclusion in the trial.

\section{STATISTICS}

\section{Between-Groups Analysis}

$E E G$. The distribution of the IBIs and SEF95 were tested for normality, if not normal, the data were transformed. For the EEG outcomes, a general univariate regression model of each EEG quantity on GA (above or below $26 \mathrm{wk}$ ) and on each of the indicators of opioid treatment, of treatment with sedative, of EEG filter, of EEG sampling rate, and of type of EEG device was conducted to see which of these quantities had a significant $(P<0.05)$ effect on the EEG outcome. The variables with significant effect on the EEG outcomes were included in multiple regression models, thereafter the estimated mean (SE) was calculated and comparisons between the two groups were conducted. 
Molecular biomarkers. If the molecular biomarkers were not normally distributed, logarithmic transformation was attempted. For data with normal distribution before or after transformation a linear mixed-model analysis using time, intervention (experimental or control), and the interaction between time and intervention was conducted. An unstructured covariance matrix was used. The analyses were adjusted by GA (above or below $26 \mathrm{wk}$ ) and centre. If the data did not follow a normal distribution, the Mann-Whitney test was used. For all analyses the two-sided $P$ values with a threshold of 0.05 were used.

\section{Inter Cluster Correlation}

The outcomes of EEG (IBI and SEF95) and the biomarker concentrations were tested for ICC to determine the correlation between the infants in each pair of twins. A mixed-model analysis was carried out for each of the EEG and biomarker outcomes: using birth (twin pair) as a random intercept. If ICC was less than 0.15 , then all infants were included in the analysis for that outcome. If the ICC was higher than 0.15 , one twin from each cluster was removed at random (using a web-based randomization program (RANDOM.ORG, Dublin, Ireland)) and the between-groups analyses were conducted on the remaining infants.

\section{Other Analyses}

The interobserver agreement for the artifact rejection of the rEEGs was estimated as proportion and the Kappa value was estimated.

The analyses were conducted using IBM SPSS Statistics for Windows version 20.0.0, (IBM SPSS, Armonk, NY) and SAS version 9.3, (SAS Institute, Cary, NC).

\section{ACKNOWLEDGMENT}

The authors would like to acknowledge Caroline Hartley for willingly sharing the Matlab script for the inter-event intervals.

\section{STATEMENT OF FINANCIAL SUPPORT}

The Danish Council for Strategic Research financially supported this work through an unconditional and unrestricted grant of DKK 11,100,105. The funder had no role in the design, conduct, or analysis of the trial.

Disclosure: None of the authors have any conflicts of interest to disclose.

\section{REFERENCES}

1. Moore T, Hennessy EM, Myles J, et al. Neurological and developmental outcome in extremely preterm children born in England in 1995 and 2006: the EPICure studies. BMJ 2012;345:e7961.

2. Volpe JJ. Brain injury in premature infants: a complex amalgam of destructive and developmental disturbances. Lancet Neurol 2009;8:110-24.

3. de Vries LS, Benders MJ, Groenendaal F. Imaging the premature brain: ultrasound or MRI? Neuroradiology 2013;55:Suppl 2:13-22.

4. Horsch S, Muentjes C, Franz A, Roll C. Ultrasound diagnosis of brain atrophy is related to neurodevelopmental outcome in preterm infants. Acta Paediatr 2005;94:1815-21.

5. Brouwer MJ, de Vries LS, Groenendaal F, et al. New reference values for the neonatal cerebral ventricles. Radiology 2012;262:224-33.

6. Wikström S, Pupp IH, Rosén I, et al. Early single-channel aEEG/EEG predicts outcome in very preterm infants. Acta Paediatr 2012;101:719-26.

7. West CR, Harding JE, Williams CE, Nolan M, Battin MR. Cot-side electroencephalography for outcome prediction in preterm infants: observational study. Arch Dis Child Fetal Neonatal Ed 2011;96:F108-13.

8. Gazzolo D, Bruschettini M, Lituania M, Serra G, Bonacci W, Michetti F. Increased urinary S100B protein as an early indicator of intraventricular hemorrhage in preterm infants: correlation with the grade of hemorrhage. Clin Chem 2001;47:1836-8.

9. Thorngren-Jerneck K, Alling C, Herbst A, Amer-Wahlin I, Marsal K. S100 protein in serum as a prognostic marker for cerebral injury in term newborn infants with hypoxic ischemic encephalopathy. Pediatr Res 2004;55:406-12.
10. Teunissen CE, Veerhuis R, De Vente J, et al. Brain-specific fatty acid-binding protein is elevated in serum of patients with dementia-related diseases. Eur J Neurol 2011;18:865-71.

11. Inder T, Mocatta T, Darlow B, Spencer C, Volpe JJ, Winterbourn C. Elevated free radical products in the cerebrospinal fluid of VLBW infants with cerebral white matter injury. Pediatr Res 2002;52:213-8.

12. Pellicer A, Greisen G, Benders M, et al. The SafeBoosC phase II randomised clinical trial: a treatment guideline for targeted near-infrared-derived cerebral tissue oxygenation versus standard treatment in extremely preterm infants. Neonatology 2013;104:171-8.

13. Hyttel-Sorensen S, Pellicer A, Alderliesten T, et al. Cerebral near infrared spectroscopy oximetry in extremely preterm infants: phase II randomised clinical trial. BMJ 2015;350:g7635.

14. Gluud C, Brok J, Gong Y, Koretz RL. Hepatology may have problems with putative surrogate outcome measures. J Hepatol 2007;46:734-42.

15. Buyse M, Molenberghs G, Paoletti X, et al. Statistical evaluation of surrogate endpoints with examples from cancer clinical trials. Biom J 2015; e-pub ahead of print February 2015

16. Molenberghs G, Burzykowski T, Alonso A, Assam P, Tilahun A, Buyse M. A unified framework for the evaluation of surrogate endpoints in mentalhealth clinical trials. Stat Methods Med Res 2010;19:205-36.

17. Inder TE, Buckland L, Williams CE, et al. Lowered electroencephalographic spectral edge frequency predicts the presence of cerebral white matter injury in premature infants. Pediatrics 2003;111:27-33.

18. Sauzet O, Wright KC, Marston L, Brocklehurst P, Peacock JL. Modelling the hierarchical structure in datasets with very small clusters: a simulation study to explore the effect of the proportion of clusters when the outcome is continuous. Stat Med 2013;32:1429-38.

19. Hartley C, Berthouze L, Mathieson SR, et al. Long-range temporal correlations in the EEG bursts of human preterm babies. PLoS One 2012;7: e31543.

20. Wikström S, Lundin F, Ley D, et al. Carbon dioxide and glucose affect electrocortical background in extremely preterm infants. Pediatrics 2011;127:e1028-34.

21. Victor S, Appleton RE, Beirne M, Marson AG, Weindling AM. Spectral analysis of electroencephalography in premature newborn infants: normal ranges. Pediatr Res 2005;57:336-41.

22. Lacey DJ, Topper WH, Buckwald S, Zorn WA, Berger PE. Preterm verylow-birth-weight neonates: relationship of EEG to intracranial hemorrhage, perinatal complications, and developmental outcome. Neurology 1986;36:1084-7.

23. Pressler RM, Boylan GB, Morton M, Binnie CD, Rennie JM. Early serial EEG in hypoxic ischaemic encephalopathy. Clin Neurophysiol 2001;112:31-7.

24. van Laerhoven H, de Haan TR, Offringa M, Post B, van der Lee JH. Prognostic tests in term neonates with hypoxic-ischemic encephalopathy: a systematic review. Pediatrics 2013;131:88-98.

25. Victor S, McKeering CM, Roberts SA, Fullwood C, Gaydecki PA. Effect of permissive hypercapnia on background cerebral electrical activity in premature babies. Pediatr Res 2014;76:184-9.

26. Fogtmann EP, Plomgaard AM, Greisen G, Gluud C. Prognostic accuracy of amplitude-integrated electroencephalogram or electroencephalogram in preterm infants: a systematic review. PROSPERO 2014. (http:// www.crd.york.ac.uk/PROSPERO_REBRANDING/display_record. asp?ID=CRD42014010514). Accessed 14 July 2014.

27. Bjerre I, Hellström-Westas L, Rosén I, Svenningsen N. Monitoring of cerebral function after severe asphyxia in infancy. Arch Dis Child 1983;58: 997-1002.

28. Iyer KK, Roberts JA, Metsäranta M, Finnigan S, Breakspear M, Vanhatalo $\mathrm{S}$. Novel features of early burst suppression predict outcome after birth asphyxia. Ann Clin Transl Neurol 2014;1:209-14.

29. Eskesen V, Springborg JB, Undén J, Romner B. Initial handling of minimal, light and moderate head traumas in adults. Ugeskr Læger 2014;176:V09130559.

30. Donato R, Sorci G, Riuzzi F, et al. S100B's double life: intracellular regulator and extracellular signal. Biochim Biophys Acta 2009;1793:1008-22. 
31. Gazzolo D, Vinesi P, Bartocci M, et al. Elevated S100 blood level as an early indicator of intraventricular hemorrhage in preterm infants. Correlation with cerebral Doppler velocimetry. J Neurol Sci 1999;170:32-5.

32. Rogers LK, Graf AE, Bhatia A, Leonhart KL, Oza-Frank R. Associations between maternal and infant morbidities and sRAGE within the first week of life in extremely preterm infants. PLoS One 2013;8:e82537.

33. Subbaswamy A, Hsu AA, Weinstein S, Bell MJ. Correlation of cerebral near-infrared spectroscopy (cNIRS) and neurological markers in critically ill children. Neurocrit Care 2009;10:129-35.

34. Wunderlich MT, Hanhoff T, Goertler M, et al. Release of brain-type and heart-type fatty acid-binding proteins in serum after acute ischaemic stroke. J Neurol 2005;252:718-24.

35. Brame CJ, Salomon RG, Morrow JD, Roberts LJ 2nd. Identification of extremely reactive gamma-ketoaldehydes (isolevuglandins) as products of the isoprostane pathway and characterization of their lysyl protein adducts. J Biol Chem 1999;274:13139-46.

36. Vesoulis ZA, Inder TE, Woodward LJ, Buse B, Vavasseur C, Mathur AM. Early electrographic seizures, brain injury, and neurodevelopmental risk in the very preterm infant. Pediatr Res 2014;75:564-9.

37. Hou X, Ding H, Teng Y, et al. Research on the relationship between brain anoxia at different regional oxygen saturations and brain damage using near-infrared spectroscopy. Physiol Meas 2007;28:1251-65.

38. Zhang D, Hou X, Liu Y, Zhou C, Luo Y, Ding H. The utility of amplitudeintegrated EEG and NIRS measurements as indices of hypoxic ischaemia in the newborn pig. Clin Neurophysiol 2012;123:1668-75.

39. Kurth CD, McCann JC, Wu J, Miles L, Loepke AW. Cerebral oxygen saturation-time threshold for hypoxic-ischemic injury in piglets. Anesth Analg 2009;108:1268-77.
40. Tin W, Gupta S. Optimum oxygen therapy in preterm babies. Arch Dis Child Fetal Neonatal Ed 2007;92:F143-7.

41. SUPPORT Study Group of the Eunice Kennedy Shriver NICHD Neonatal Research Network. Target ranges of oxygen saturation in extremely preterm infants. N Engl J Med 2010;362:1959-69.

42. Hyttel-Sorensen S, Austin T, van Bel F, et al. A phase II randomized clinical trial on cerebral near-infrared spectroscopy plus a treatment guideline versus treatment as usual for extremely preterm infants during the first three days of life (SafeBoosC): study protocol for a randomized controlled trial. Trials 2013;14:120.

43. O'Reilly D, Navakatikyan MA, Filip M, Greene D, Van Marter LJ. Peak-topeak amplitude in neonatal brain monitoring of premature infants. Clin Neurophysiol 2012;123:2139-53.

(c) (1) $(\Theta$ This work is licensed under a Creative Commons Attribution-NonCommercial-NoDerivs $\quad 4.0$ International License. The images or other third party material in this article are included in the article's Creative Commons license, unless indicated otherwise in the credit line; if the material is not included under the Creative Commons license, users will need to obtain permission from the license holder to reproduce the material. To view a copy of this license, visit http://creativecommons.org/licenses/by-nc-nd/4.0/ 